\title{
Pion crystals hosting topologically stable baryons
}

\author{
Fabrizio Canfora, ${ }^{1, *}$ Stefano Carignano $\odot^{2, \dagger}$ Marcela Lagos, ${ }^{3, \star}$ Massimo Mannarelli ${ }^{4, \S}$ and Aldo Vera $\oplus^{3, \|}$ \\ ${ }^{1}$ Centro de Estudios Científicos (CECS), Casilla 1469, Valdivia, Chile \\ ${ }^{2}$ Departament de Física Quàntica i Astrofísica and Institut de Ciències del Cosmos, \\ Universitat de Barcelona, Martí i Franquès 1, 08028 Barcelona, Catalonia, Spain \\ ${ }^{3}$ Instituto de Ciencias Físicas y Matemáticas, Universidad Austral de Chile, Casilla 567, Valdivia, Chile \\ ${ }^{4}$ INFN, Laboratori Nazionali del Gran Sasso, Via G. Acitelli, 22, I-67100 Assergi (AQ), Italy
}

(Received 21 December 2020; accepted 8 March 2021; published 5 April 2021)

\begin{abstract}
We construct analytic $(3+1)$-dimensional inhomogeneous and topologically nontrivial pion systems using chiral perturbation theory. We discuss the effect of isospin asymmetry with vanishing electromagnetic interactions as well as some particular configurations with nonvanishing electromagnetic interactions. The inhomogeneous configurations of the pion fields are characterized by a nonvanishing topological charge that can be identified with baryons surrounded by a cloud of pions. This system supports a topologically protected persistent superflow. When the electromagnetic field is turned on the superflow corresponds to an electromagnetic supercurrent.
\end{abstract}

DOI: 10.1103/PhysRevD.103.076003

\section{INTRODUCTION}

One of the main goals of the theoretical and experimental investigations in quantum chromodynamics (QCD) is to determine the phases of hadronic matter as a function of temperature, baryonic density, and isospin asymmetry. In the grand canonical ensemble this amounts to studying the realization of the hadronic phases as a function of the baryonic chemical potential, $\mu_{B}$, which encodes the baryonic density, and the isospin chemical potential, $\mu_{I}$, which determines the isospin asymmetry.

Since QCD is an asymptotically free theory we expect that at some large energy scale hadrons melt, liberating their quark and gluon content [1]. High temperature deconfined hadronic matter has been realized in relativistic heavy-ion colliders (see, for instance, [2-4]), and it can possibly form in the core of compact stars $[5,6]$. In any terrestrial heavy-ion experiment, as well as in the core of compact stars, QCD is in the nonperturbative regime, posing a number of challenging problems to the determination of the matter properties (see [7] for a review). In order to get insight on the properties of hadronic matter,

\footnotetext{
*anfora@cecs.cl

†stefano.carignano@fqa.ub.edu

\#marcela.lagos@uach.cl

\$massimo@lngs.infn.it

aldo.vera@uach.cl
}

Published by the American Physical Society under the terms of the Creative Commons Attribution 4.0 International license. Further distribution of this work must maintain attribution to the author(s) and the published article's title, journal citation, and DOI. Funded by SCOAP. many different methods have been developed. At vanishing baryonic density the deconfined phase can be studied by lattice QCD (LQCD) methods [8,9], but with increasing baryonic density these numerical simulations become problematic; they are hampered by the so-called sign problem (see [10-14] for recent progress in this direction). For vanishing baryonic density and up to $\mu_{I} \simeq 2 m_{\pi}$, LQCD simulations are feasible [15-29] and their results can be compared with those obtained by chiral perturbation theory $(\chi \mathrm{PT})$ [30-55], or by Nambu-Jona-Lasinio models [56-81]. In this way, one can probe the robustness of the obtained results. In particular, it is now well established that when the isospin chemical potential exceeds the pion mass there is a second order phase transition between the normal phase and the pion condensed phase (see [82] for a recent review).

Various regions of the QCD phase diagram may be occupied by inhomogeneous phases (see, for instance, [83-88]). The analysis of models in the $(1+1)$ and $(3+1)$ dimensions has shown that at low temperatures some crystalline structures can be thermodynamically stable and energetically favored with respect to the homogeneous phase. A quite relevant result in this area has been the construction of exact crystalline solutions of ordered solitons (see [89-97]). Whether an ensemble of charged pions may form an inhomogeneous Bose-Einstein condensate at sufficiently low temperature is an interesting possibility [98,99]. An example of an inhomogeneous phase is the chiral soliton lattice, which is an inhomogeneous pionic phase supported by strong external fields $[100,101]$. In these works the order parameter depends on only one spatial coordinate, allowing in this way the use of 
tools developed in Gross-Neveu models [102-105]. This fact, however, prevents the condensate itself from having a nontrivial topological charge.

Topological stability can be achieved in $(3+1)$ dimensional inhomogeneous condensates. However, a detailed analysis of the electromagnetic interactions of $(3+1)$-dimensional spatially modulated condensates is not easy; in these situations the only available numerical results on crystals of solitons treat the electromagnetic field as a fixed external field neglecting the back reaction of the hadronic matter. It would be an extremely useful result to achieve a sound analytic control on gauged solitons with high topological charge and with crystalline order. Explicit examples have been obtained either in lower dimensions or when some extra symmetries (such as SUSY) are available (see [106-114]).

In the present paper we use zero temperature gauged twoflavor $\chi \mathrm{PT}$ to construct an analytic $(3+1)$-dimensional pion inhomogeneous condensate characterized by a nonvanishing topological charge. We achieve this result by an appropriate choice of the condensate ansatz and of the gauge field configuration. The presence of a topological charge prevents the decay of the inhomogeneous condensate into a homogeneous phase, but it is not a sufficient condition for stability. A classical argument by Landau and Peierls is that in three or fewer dimensions the thermal fluctuations destroy the condensates depending on only one spatial coordinate [115]. This is the reason why we consider a $(3+1)$ dimensional modulation, thus corresponding to a crystallinelike phase. Regarding the stability of these kinds of models, Skyrme and Derrick showed [116-119] that they do not support static solitonic solutions in flat, topologically trivial $(3+1)$-dimensional space-time. We will circumvent this argument by considering a finite spatial volume, as finite volume effects, together with nontrivial boundary conditions at finite volume, break Derrick's scaling argument [119]. These ways to avoid the Derrick no-go argument will be combined using the generalized hedgehoglike ansatz introduced in [120-138] that we will properly extend at nonvanishing isospin chemical potential. In order to construct topologically stable solitons, the previous works [120-138] needed to consider a time dependent modulation with time dependent boundary conditions. Within the present work we show that for the nonvanishing isospin chemical potential, it is possible to obtain a topologically stable crystalline phase with a static background field and with time independent boundary conditions. This implies that the proposed crystalline phase may be studied in LQCD simulations, which employ static boundary conditions.

Regarding the use of $\chi \mathrm{PT}$, we remark that it is quantitatively under control for $\mu_{I}<\Lambda_{c} \sim 1 \mathrm{GeV}$, corresponding to the critical scale of $\chi \mathrm{PT}$. This effective field theory is based on two key ingredients: the global symmetries of QCD and an appropriate low momentum expansion
[139-147]. The results obtained within $\chi \mathrm{PT}$ agree with those of other methods for $\mu_{I} \leq 2 m_{\pi}$ (see the discussion in [82]), corroborating the reliability of this effective field theory. Remarkably, $\chi$ PT can also be used to study a variety of gauge theories with isospin asymmetry, including two color QCD with different flavors [148-154].

It is worth emphasizing that, while we shall study the inhomogeneous condensates in the context of $\chi$ PT, the results reported in [136-138] strongly suggest that the existence of such condensates (as well as their explicit functional forms) are very robust. In particular, the construction is not spoiled either by subleading corrections in the 't Hooft expansion or by replacing the $S U(2)$ internal symmetry with an $S U(N)$ internal symmetry group. Hence, it is very natural to think that the results obtained in the present manuscript could be valid even beyond $\chi \mathrm{PT}$; we hope to come back on this very interesting issue in a future publication.

This paper is organized as follows. In Sec. II we briefly review the application of $\chi \mathrm{PT}$ to meson condensation. In Sec. III we discuss the inhomogeneous pion phase for vanishing electromagnetic fields. In Sec. IV we consider the gauged model with a particular configuration of the gauge fields. We draw our conclusions in Sec. V.

We use the Minkowski metric $\eta_{\mu \nu}=(1,-1,-1,-1)$ and the natural units $c=\hbar=1$.

\section{THE $\chi$ PT DESCRIPTION OF MESON CONDENSATION}

The low-energy properties of pions can be described by $\chi$ PT [139-147], which is grounded on the global symmetries of QCD and uses an expansion in exchanged momenta. In this approach the pion fields can be collected in the unimodular field,

$$
\Sigma=e^{i \cdot \sigma}=\mathbf{1}_{2} \cos \alpha \pm i N \sin \alpha,
$$

where $N=n \cdot \sigma$, with $\sigma$ being the Pauli matrices, and $\alpha=\alpha n$; we shall call $\alpha$ the radial field while $n$ is a unimodular field in isospin space. This is a convenient representation because it allows us to simplify the calculations; we shall see below how these fields are related to the standard pion fields. The leading order $S U(2) \chi \mathrm{PT}$ Lagrangian including the electromagnetic interaction can be written as

$$
\mathcal{L}=\frac{f_{\pi}^{2}}{4} \operatorname{Tr}\left[-\left(\Sigma^{\mu} \Sigma_{\mu}\right)+m_{\pi}^{2}\left(\Sigma+\Sigma^{\dagger}\right)\right]-\frac{1}{4} F_{\mu \nu} F^{\mu \nu},
$$

where the pion decay constant, $f_{\pi} \simeq 93 \mathrm{MeV}$, and the assumed degenerate pion masses, $m_{\pi} \simeq 135 \mathrm{MeV}$, are phenomenological constants. Also,

$$
\Sigma_{\mu}=\Sigma^{-1} D_{\mu} \Sigma=\Sigma_{\mu}^{j} \sigma_{j}
$$


The field strength is $F_{\mu \nu}=\partial_{\mu} A_{\nu}-\partial_{\nu} A_{\mu}$, where $A^{\mu}$ is the electromagnetic gauge field and the covariant derivative is defined as

$$
D_{\mu} \Sigma=\partial_{\mu} \Sigma+i \tilde{A}_{\mu}\left[\sigma_{3}, \Sigma\right]
$$

where $\partial_{\mu}$ is the usual partial derivative and

$$
\tilde{A}^{\mu}=\left(\frac{\mu_{I}}{2}+A^{0}, \boldsymbol{A}\right),
$$

where we have included the effect of the isospin chemical potential, $\mu_{I}$. Regarding the gauge field potential, we shall assume that it is self-consistently generated by the pion distribution. The classical field equations read

$$
\begin{gathered}
D_{\mu} \Sigma^{\mu}+\frac{m_{\pi}^{2}}{2}\left(\Sigma-\Sigma^{\dagger}\right)=0, \\
\partial_{\mu} F^{\mu \nu}=J^{\nu},
\end{gathered}
$$

where

$$
J^{\mu}=i \frac{f_{\pi}^{2}}{2} \operatorname{Tr}\left[\Sigma^{\mu}\left(\Sigma^{\dagger} \sigma_{3} \Sigma-\sigma_{3}\right)\right]
$$

is the pion current generated by the electromagnetic field.

To make contact with the usual pion representation we expand Eq. (1), retaining the leading order in $\alpha$. In this way, Eq. (6) yields

$$
\left(D_{\mu} D^{\mu}+m_{\pi}^{2}\right) \alpha=0
$$

which is the Klein-Gordon equation for three scalar fields. This equation can be diagonalized to

$$
\begin{gathered}
\left(\partial_{\mu} \partial^{\mu}+m_{\pi}^{2}\right) \alpha_{3}=0, \\
\left(\partial_{\mu} \partial^{\mu}+4 i \tilde{A}^{\mu} \partial_{\mu}+m_{\pi}^{2}-4 \tilde{A}^{\mu} \tilde{A}_{\mu}\right)\left(\alpha_{1}+i \alpha_{2}\right)=0, \\
\left(\partial_{\mu} \partial^{\mu}-4 i \tilde{A}^{\mu} \partial_{\mu}+m_{\pi}^{2}-4 \tilde{A}^{\mu} \tilde{A}_{\mu}\right)\left(\alpha_{1}-i \alpha_{2}\right)=0,
\end{gathered}
$$

which explicitly shows that $\alpha_{3}$ is the neutral field, while $\pi_{ \pm} \propto\left(\alpha_{1} \pm i \alpha_{2}\right)$ correspond to the two charged pion fields. For vanishing electromagnetic potential, the charged scalar fields have dispersion law

$$
E_{ \pm}= \pm \mu_{I} \pm \sqrt{p^{2}+m_{\pi}^{2}}
$$

which manifestly shows that for $\left|\mu_{I}\right|=m_{\pi}$ a massless mode appears, signaling a transition to the homogeneous pion condensed phase.

\section{A. The homogeneous phase}

Let us briefly recall the most important results of the two-flavor homogeneous and time independent pion condensed phase. This phase is characterized by the condensation of one of the two charged pion fields, which induces the spontaneous symmetry breaking

$$
\underbrace{U(1)_{I} \times U(1)_{Y}}_{\supset U(1)_{Q}} \times U(1)_{B} \rightarrow \underbrace{U(1)_{Y} \times U(1)_{B}}_{\not \supset U(1)_{Q}},
$$

where $U(1)_{I}, U(1)_{Y}$, and $U(1)_{B}$ are three unitary groups associated with the third component of isospin, hypercharge, and baryonic number, respectively (see [82]), whereas $U(1)_{Q}$ is the gauge group of the electromagnetic interaction. The condensation happens at $\left|\mu_{I}\right|=m_{\pi}$, where one of the pion modes becomes massless [see Eq. (13)]. This mode corresponds to the Nambu-Goldston boson (NGB) associated with the spontaneous $U(1)_{I}$ breaking. Neglecting the electromagnetic interaction, the broken phase is a superfluid, while it is an electromagnetic superconductor if the symmetry group is gauged.

In $\chi \mathrm{PT}$ the pion condensate can be introduced, assuming that the unimodular field $\Sigma$ in Eq. (1) takes a nontrivial vev, $\bar{\Sigma}$, which can be determined by treating $\alpha$ and $N$ as variational parameters. The most general ansatz for the unit vector background field is

$$
n^{1}=\sin \Theta \cos \Phi, n^{2}=\sin \Theta \sin \Phi, n^{3}=\cos \Theta,
$$

where $\Theta$ and $\Phi$ are two variational angles.

Upon substituting this vev in Eq. (2), one obtains [40]

$\mathcal{L}_{\text {hom }}=f_{\pi}^{2} m_{\pi}^{2} \cos \alpha+2 f_{\pi}^{2} \sin ^{2} \alpha \tilde{A}^{\mu} \tilde{A}_{\mu} \sin ^{2} \Theta-\frac{1}{4} F_{\mu \nu} F^{\mu \nu}$,

which has the following well known feature: The normal phase is stable only for $\left|\mu_{I}\right|<m_{\pi}$. In this case, $\cos \alpha=1$, thus $\bar{\Sigma}=\operatorname{diag}(1,1)$, while $\Theta$ and $\Phi$ are undetermined. The associated vacuum pressure and energy density are, respectively, given by

$$
p_{\mathrm{N}}=f_{\pi}^{2} m_{\pi}^{2}, \quad \epsilon_{\mathrm{N}}=-p_{\mathrm{N}},
$$

while the number density is zero.

When $\left|\mu_{I}\right|>m_{\pi}$, the system makes a second order phase transition to the homogeneous charged pion condensed phase. In this case the trivial vacuum is unstable and the energetically favored phase is characterized by

$$
\cos \alpha_{0}=\frac{m_{\pi}^{2}}{\mu_{I}^{2}}, \quad \Theta_{0}=\frac{\pi}{2},
$$

while $\Phi$ can take any arbitrary value. Since the static Lagrangian in Eq. (16) does not depend on $\Phi$, the potential has a flat direction orthogonal to the 3-direction in isospin 
space which is spanned by the NGB. In the broken phase, the normalized pressure and the energy density (obtained by subtracting the vacuum values), are respectively given by

$$
\begin{gathered}
p=\frac{f_{\pi}^{2}}{2 \mu_{I}^{2}}\left(\mu_{I}^{2}-m_{\pi}^{2}\right)^{2}, \\
\epsilon=\frac{f_{\pi}^{2}}{2 \mu_{I}^{2}}\left(\mu_{I}^{2}-m_{\pi}^{2}\right)\left(\mu_{I}^{2}+3 m_{\pi}^{2}\right),
\end{gathered}
$$

which are positive and vanish at the phase transition point.

The aspects of the homogeneous phase that will be relevant in the discussion of the inhomogeneous phases are the following: In the broken phase the value of the radial angle $\alpha$ depends on $\mu_{I}^{2}$ by Eq. (18), while the normal phase is characterized by $\alpha=2 k \pi$, with $k$ being an integer. For $k=1, \alpha$ can only assume values in the intervals $[0, \pi / 2]$ and $[3 \pi / 2,2 \pi]$; values outside these intervals cannot be attained in the homogeneous phase. The angle $\Theta$ is not specified in the unbroken phase, but equals $\pi / 2$ in the broken phase. The flat direction of the potential corresponds to the one orthogonal to $n_{3}$ and spanned by the angle $\Phi$. Finally, in the broken phase any electromagnetic field is screened, as indicated by the second term on the right-hand-side of Eq. (16), meaning that supercurrents can circulate with vanishing resistance.

\section{THE INHOMOGENEOUS TOPOLOGICAL PHASES FOR VANISHING GAUGE FIELDS}

We begin with studying the inhomogeneous phases with vanishing gauge fields. For the appearance of the inhomogeneous topological phases, finite volume effects are of crucial importance. We take them into account using the following metric:

$$
d s^{2}=d t^{2}-\ell^{2}\left(d r^{2}+d \theta^{2}+d \phi^{2}\right)
$$

where

$$
\ell=\frac{b}{m_{\pi}}
$$

where $b$ is a real number and is the typical dimension of the system. With this coordinate choice the derivative operator turns to $\partial_{\mu}=\left(\frac{\partial}{\partial t}, \frac{1}{\ell} \frac{\partial}{\partial r}, \frac{1}{\ell} \frac{\partial}{\partial \theta}, \frac{1}{\ell} \frac{\partial}{\partial \phi}\right)$. The adimensional coordinates $r, \theta$, and $\phi$ have the ranges

$$
0 \leq r \leq 2 \pi, \quad 0 \leq \theta \leq \pi, \quad 0 \leq \phi \leq 2 \pi,
$$

meaning that we are considering pions in a cell of volume $4 \pi^{3} \ell^{3}$. For the ground state solution we assume the unimodular form of Eq. (1) that is now promoted to be a classical field, meaning that $\alpha \equiv \alpha\left(x^{\mu}\right), \Theta \equiv \Theta\left(x^{\mu}\right)$, and
$\Phi \equiv \Phi\left(x^{\mu}\right)$, with appropriate Dirichlet boundary conditions. In particular, we demand that

$n(t, r, 0, \phi)=-n(t, r, \pi, \phi), \quad n(t, r, \theta, 0)=n(t, r, \theta, 2 \pi)$,

and that

$$
\Sigma(t, 0, \theta, \phi)= \pm \Sigma(t, 2 \pi, \theta, \phi) .
$$

As we will see, these boundary conditions allow us to have a nonvanishing topological charge. Different boundary conditions can be accordingly implemented.

For vanishing electromagnetic fields, the matter effective Lagrangian in Eq. (2) can be rewritten as

$$
\begin{aligned}
\mathcal{L}_{\mathrm{m}}= & \frac{f_{\pi}^{2}}{2}\left[\partial_{\mu} \alpha \partial^{\mu} \alpha+\sin ^{2} \alpha \partial_{\mu} \Theta \partial^{\mu} \Theta+2 m_{\pi}^{2} \cos \alpha\right. \\
& \left.+\sin ^{2} \alpha \sin ^{2} \Theta\left(\partial_{\mu} \Phi-\mu_{I} \delta_{\mu 0}\right)\left(\partial^{\mu} \Phi-\mu_{I} \delta^{\mu 0}\right)\right],
\end{aligned}
$$

showing that the three classical fields $\alpha, \Theta$, and $\Phi$ are nonlinearly interacting. Solving the classical problem amounts to finding the solutions of Eqs. (6) and (7) with a vanishing gauge field, which is equivalent to solving the three coupled differential equations:

$$
\begin{gathered}
\partial_{\mu} \partial^{\mu} \Phi=-\left(\partial_{\mu} \Phi-\mu_{I} \delta_{\mu_{0}}\right) \partial^{\mu}\left(\log \left(\sin ^{2} \alpha \sin ^{2} \Theta\right)\right), \\
\partial_{\mu} \partial^{\mu} \Theta=-2 \cot \alpha \partial^{\mu} \Theta \partial_{\mu} \alpha+\frac{\sin 2 \Theta}{2} K, \\
\partial_{\mu} \partial^{\mu} \alpha=-m_{\pi}^{2} \sin \alpha+\frac{\sin (2 \alpha)}{2}\left(\partial_{\mu} \Theta \partial^{\mu} \Theta+K \sin ^{2} \Theta\right),
\end{gathered}
$$

where $K=\left(\partial_{\mu} \Phi-\mu_{I} \delta_{\mu_{0}}\right)\left(\partial^{\mu} \Phi-\mu_{I} \delta^{\mu_{0}}\right)$, which is a nontrivial task. From the discussion of the homogeneous phase, we expect that the $\Phi$ field corresponds to an NGB. Indeed, in the above equations $\Phi$ is the only field that is massless and with derivative interactions, as appropriate for an NGB. This is more evident assuming that $\Phi \equiv \Phi(t, \phi)$ and that $\alpha$ and $\Theta$ depend only on $r$ and $\theta$. Then, Eq. (27) simplifies to the free field equation of a massless scalar field,

$$
\partial_{\mu} \partial^{\mu} \Phi=\left(\frac{\partial^{2}}{\partial t^{2}}-\frac{1}{\ell^{2}} \frac{\partial^{2}}{\partial \phi^{2}}\right) \Phi=0,
$$

which has the standard free field propagating solution. However, we are interested in solitonic solutions, and therefore we shall consider the solution of Eq. (30) that depends linearly on $t$ and $\phi$ of the form

$$
\Phi=\frac{a}{\ell} t-p \phi+\Phi_{0}
$$

where $a$ and $\Phi_{0}$ are real numbers and $p \in \mathbb{Z}$. In this way, Eqs. (28) and (29) yield 


$$
\begin{aligned}
\nabla^{2} \Theta= & -2 \cot \alpha\left(\partial_{r} \Theta \partial_{r} \alpha+\partial_{\theta} \Theta \partial_{\theta} \alpha\right)-K \ell^{2} \frac{\sin 2 \Theta}{2}, \\
\nabla^{2} \alpha= & \frac{1}{2} \sin (2 \alpha)\left(\left(\partial_{\theta} \Theta\right)^{2}+\left(\partial_{r} \Theta\right)^{2}-K \ell^{2} \sin ^{2}(\Theta)\right) \\
& +m_{\pi}^{2} \ell^{2} \sin \alpha,
\end{aligned}
$$

where $\nabla^{2}=\left(\frac{\partial^{2}}{\partial \theta^{2}}+\frac{\partial^{2}}{\partial r^{2}}\right)$, and

$$
K=\left(\frac{a}{\ell}-\mu_{I}\right)^{2}-\frac{p^{2}}{\ell^{2}}
$$

is now a constant. This system of equations is still complicated, however we can obtain analytical solutions in some particular cases.

If $\alpha$ is a constant then Eq. (32) becomes a sine-Gordonlike equation with solution

$$
\Theta(\bar{\theta}, \bar{r})=2 \arctan \left[\frac{\sinh \left(w \bar{\theta} / \sqrt{w^{2}-1}\right)}{w \cos \left(\bar{r} / \sqrt{w^{2}-1}\right)}+\delta\right],
$$

where $\bar{r}=\ell r K / 2$ and $\bar{\theta}=\ell \theta K / 2$, while $w$ and $\delta$ are two constants that depend on the boundary conditions. Asking that $\Theta(0, \bar{r})=0$, we readily fix $\delta=0$. Then, according to Eq. (24), we should demand that for any $\bar{r}$, $\Theta(0, \bar{r})=(2 k+1) \pi$, with $k$ integer. However, this is not compatible with Eq. (35). Moreover, the only solution with $\alpha$ constant of Eq. (33) is $\alpha=n \pi$ with $n$ integer, corresponding to the homogeneous normal phase. For these reasons we shall not consider this solution anymore.

A different class of solutions can be obtained considering $\Theta \equiv \Theta(\theta)$ and $\alpha \equiv \alpha(r)$. In order to make Eq. (33) independent of $\theta$ we have to assume that $\Theta$ depends linearly on $\theta$ and that $K=0$. It follows that in this case, the unit vector in Eq. (15) is modulated as

$$
\Phi=\frac{a}{\ell} t-p \phi+\Phi_{0}, \quad \Theta=q \theta+\Theta_{0},
$$

where $a, \Theta_{0}$, and $\Phi_{0}$ are real numbers, $p, q \in \mathbb{Z}$ with odd $q$. As remarked in [120-138], the time dependence of the unit vector is sufficient to avoid the Derrick's no-go theorem on the existence of solitons in nonlinear scalar field theories. It corresponds to a unit vector rotating at constant speed around the 3-direction in isospin space, which is precisely the flat potential direction discussed in the homogeneous phase. However, this is not a necessary condition; indeed the considered system is confined in a finite volume and this suffices to avoid the scaling argument of Derrick's theorem. It remains to impose the condition $K=0$, which can be written as

$$
a=\ell \mu_{I}+p
$$

where $p$ can be a positive or a negative integer. This condition relates the parameter of the classical field $\Phi$ with the isospin chemical potential. Remarkably, it is possible to eliminate any time dependence; imposing that $a=0$ it follows that

$$
p=-\mu_{I} \ell,
$$

which relates in a clear way the finite volume size and the isospin chemical potential. Notice that it is possible to eliminate the time dependence only for nonvanishing isospin chemical potentials. The advantage of this choice is that the boundary conditions at $\phi=0$ and $\phi=2 \pi$ become time independent and can be possibly implemented in LQCD simulations. Finally, the $\theta$ dependence of the $\Theta$ field allows us to span all its possible values, including the one in Eq. (18), corresponding to the maximum of the homogeneous Lagrangian. Upon substituting Eq. (36) in the differential equation (29), one readily finds that it can be written as [135]

$$
\frac{\partial^{2} \alpha}{\partial r^{2}}=m_{\pi}^{2} \ell^{2} \sin \alpha+\frac{q^{2}}{2} \sin (2 \alpha)
$$

meaning that the modulation of the $\alpha$ field does not explicitly depend on the isospin chemical potential. This seems at odds with the result of the homogeneous broken phase in Eq. (18). However, $\ell$ and $\mu_{I}$ are related by Eq. (38), therefore there is an implicit dependence on $\mu_{I}$. It is indeed possible to obtain the homogeneous solution from Eq. (39), noticing that in this case it gives

$$
\cos \bar{\alpha}=-\frac{m_{\pi}^{2}}{\mu_{I}^{2}}\left(\frac{p}{q}\right)^{2}
$$

which is indeed similar to Eq. (18). Therefore, the homogeneous phase corresponds to the prescription

$$
p= \pm q, \quad \alpha_{0}=\bar{\alpha}+\pi \text {. }
$$

Regarding the general $\alpha(r)$ modulation, the radial field second order differential equation (39) can be recast as the first order differential equation

$$
\frac{\partial \alpha}{\partial r}=\eta(\alpha)
$$

where we can determine the function $\eta(\alpha)$ noticing that

$$
\frac{\partial^{2} \alpha}{\partial r^{2}}=\frac{\partial \eta}{\partial r}=\frac{1}{2} \frac{\partial \eta^{2}}{\partial \alpha}
$$

Then from Eq. (39) we obtain

$$
\eta(\alpha)= \pm \sqrt{\eta_{0}^{2}+2 m_{\pi}^{2} \ell^{2}(1-\cos \alpha)+q^{2} \sin ^{2}(\alpha)},
$$

where $\eta_{0}$ is an adimensional integration constant and the positive (negative) sign corresponds to solutions with 


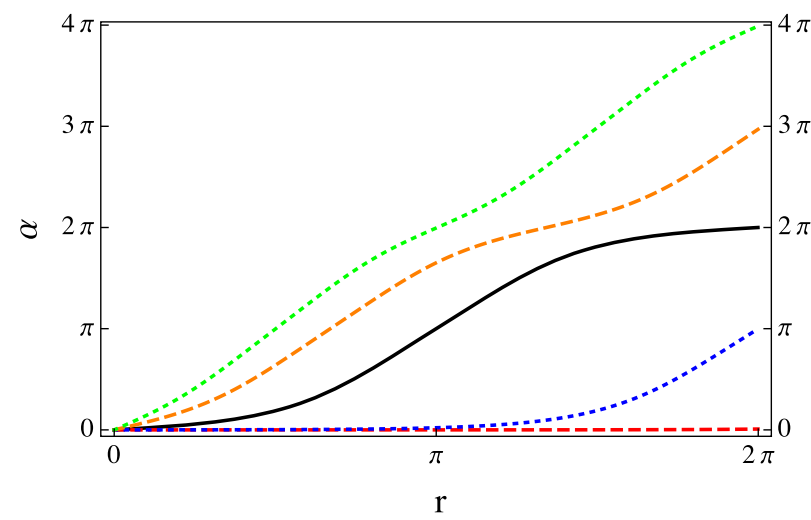

FIG. 1. Modulation of the radial mode, $\alpha(r)$, obtained numerically by solving Eq. (42) for $q=1$ and $\ell=1 / m_{\pi}$, assuming $\alpha(0)=0$. We report the results obtained with five different values of the integration constant $\eta_{0}$ [see Eq. (44)] to match the right boundary condition $\alpha(2 \pi)=n \pi$. For $\eta_{0}=0$ (red dashed line), the radial mode identically vanishes. With nonvanishing values of $\eta_{0}$ the radial field monotonically grows and the right boundary value, $\alpha(2 \pi)$, increases with increasing values of $\eta_{0}$.

increasing (decreasing) values of $\alpha(r)$ in the interval $r \in[0,2 \pi]$. In the following we shall assume that $\eta$ is non-negative and that $\eta_{0}$ is such that for a given $q$,

$$
\int_{0}^{n \pi} \frac{d \alpha}{\eta(\alpha)}=2 \pi
$$

where we have assumed the boundary conditions

$$
\alpha(0)=0 \quad \text { and } \quad \alpha(2 \pi)=n \pi,
$$

where $n$ is an integer. Even (odd) values of $n$ correspond to periodic (antiperiodic) boundary conditions in the $r$ direction [see Eq. (25)]. The above integral can be evaluated in terms of elliptic functions; alternatively, one can fix $\eta_{0}$ by

$$
n \pi=\alpha(2 \pi)=\int_{0}^{2 \pi} d r \eta(\alpha(r)),
$$

which follows from Eq. (42). Therefore, for a given value of $q$ and $\ell$, the integration constant $\eta_{0}$, determines the value of the $\alpha$ field at the right boundary, which, as we shall see, is linked to the topological charge $[155,156]$. We report in Fig. 1 the plot of the radial field as a function of $r$ for $q=1$ and $\ell=1 / m_{\pi}$, corresponding to $b=1$ in Eq. (22) and five different values of $\eta_{0}$, corresponding to the boundary condition in Eq. (46) with $n=0,1,2,3$, 4. For $\eta_{0}=0$ (red dashed line), the $\alpha$ field identically vanishes. This case corresponds to the homogeneous normal phase. With increasing $\eta_{0}$, the value of $\alpha$ at the right boundary increases. The values $n=\{1,2,3,4\}$ are respectively obtained with $\eta_{0} \simeq\{0.002,0.186,0.74,1.38\}$. With reference to the solid black line, we notice that the $\alpha$ field assumes all the possible values in the $[0,2 \pi]$ interval, while

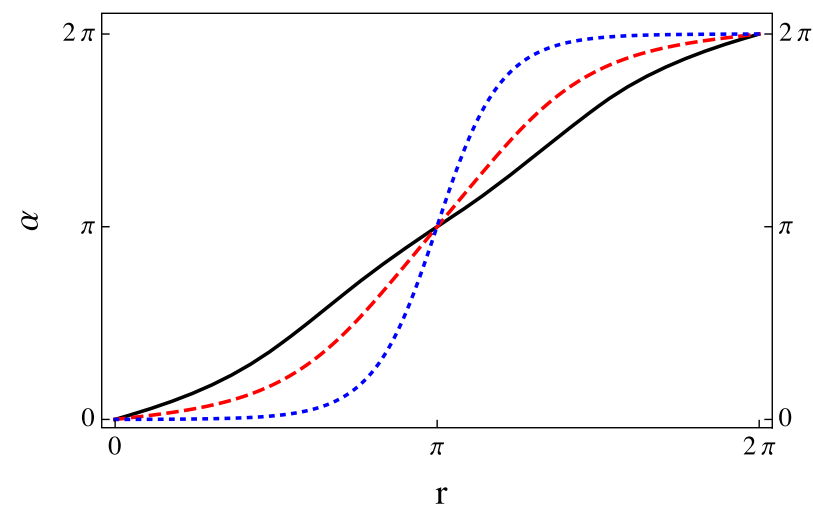

FIG. 2. Modulation of the radial mode, $\alpha(r)$, obtained numerically by solving Eq. (42) for three different values of the lattice size [see Eq. (22)]. The solid black line corresponds to $b=0.5$, the dashed red line to $b=1$, and the dotted blue line to $b=2.5$.

in the homogeneous phase it can only assume values in the intervals $[0, \pi / 2]$ and $[3 \pi / 2,2 \pi]$. With increasing values of the lattice size, the shape of the $\alpha$ field changes. In Fig. 2 we show the plot of the $\alpha$ field for $n=2$ and three different values of $b$. With increasing values of $b$ the modulation tends to become steeper at $r=\pi$ and flattens at the boundary. For large values of $b$ the system tends to the homogeneous normal phase: the integration constant $\eta_{0}$ decreases with increasing system size and eventually vanishes for asymptotic values of $b$. We have seen above that for $\eta_{0}=0$ one obtains the homogeneous normal phase, however, in this case we have imposed that $\alpha(2 \pi)=2 \pi$, therefore the $\alpha$ field discontinuously jumps from 0 to $2 \pi$ at $r=\pi$. The fact that the large size case corresponds to the homogeneous normal phase can be seen by combining Eqs. (22) and (38) in

$$
\frac{\mu_{I}}{m_{\pi}}=-\frac{p}{b}
$$

and therefore asymptotic values of $b$ correspond to vanishing $\mu_{I}$. For numerical evaluations, we shall hereafter assume the values

$q=1, \quad p=1, \quad \ell=1 / m_{\pi}, \quad \eta_{0} \simeq 0.186$,

where the last equation implies that $\alpha(2 \pi)=2 \pi$.

\section{A. The energy-momentum tensor}

For a given Lagrangian density, $\mathcal{L}$, the energymomentum tensor is

$$
T_{\mu \nu}=2 \frac{\partial \mathcal{L}}{\partial g^{\mu \nu}}-g_{\mu \nu} \mathcal{L},
$$

and using the expression in Eq. (26) we obtain the matter contribution 


$$
\begin{aligned}
T_{\mu \nu}^{\mathrm{m}}= & -\frac{f_{\pi}^{2}}{2} \operatorname{Tr}\left(\Sigma_{\mu} \Sigma_{\nu}\right)+\frac{f_{\pi}^{2}}{4} g_{\mu \nu} \operatorname{Tr}\left(\Sigma^{\alpha} \Sigma_{\alpha}\right) \\
& -g_{\mu \nu} f_{\pi}^{2} m_{\pi}^{2} \cos \alpha .
\end{aligned}
$$

Upon substituting Eq. (36) in Eq. (51) and normalizing by subtracting the vacuum energy density [see Eq. (17)], we obtain the matter energy density

$$
\begin{aligned}
\epsilon_{\mathrm{m}}=T_{00}^{\mathrm{m}}+f_{\pi}^{2} m_{\pi}^{2}= & \frac{f_{\pi}^{2}}{2 \ell^{2}}\left(\eta^{2}+2 m_{\pi}^{2} \ell^{2}(1-\cos \alpha)\right. \\
& \left.+\left(2 p^{2} \sin ^{2}(q \theta)+q^{2}\right) \sin ^{2} \alpha\right) .
\end{aligned}
$$

Taking into account the ranges in Eq. (23), the total energy of the system is

$$
E_{\mathrm{m}}=\ell^{3} \int d r d \theta d \phi \epsilon_{\mathrm{m}}=\pi^{2} f_{\pi}^{2} \ell \int_{0}^{2 \pi} \Omega(\alpha) d \alpha,
$$

where

$$
\Omega(\alpha)=\eta(\alpha)+\left(q^{2}+p^{2}\right) \frac{\sin ^{2}(\alpha)}{\eta(\alpha)}+2 m_{\pi}^{2} \ell^{2} \frac{1-\cos (\alpha)}{\eta(\alpha)},
$$

depends on the numerical values of the various constants. For the particular choice in Eq. (49) we obtain

$$
E_{\mathrm{m}}=20.5 \pi^{2} \frac{f_{\pi}^{2}}{m_{\pi}} .
$$

The components of the pressure are instead given by

$$
\begin{aligned}
P_{r r}= & \frac{f_{\pi}^{2}}{2 \ell^{2}}\left(\eta^{2}-q^{2} \sin ^{2} \alpha+2 m_{\pi}^{2} \ell^{2} \cos \alpha\right), \\
P_{\theta \theta}= & \frac{f_{\pi}^{2}}{2 \ell^{2}}\left(-\eta^{2}+q^{2} \sin ^{2} \alpha+2 m_{\pi}^{2} \ell^{2} \cos \alpha\right), \\
P_{\phi \phi}= & \frac{f_{\pi}^{2}}{2 \ell^{2}}\left(2 \ell^{2} m_{\pi}^{2} \cos \alpha-\eta^{2}-q^{2} \sin ^{2} \alpha\right. \\
& \left.+2 \sin ^{2}(\alpha) \sin ^{2}(q \theta) p^{2}\right),
\end{aligned}
$$

which show that the pressure is not isotropic. This happens because of the space modulation of the $\alpha$ and $\Theta$ fields. When substituting Eq. (44) in Eq. (55), we obtain

$$
\begin{aligned}
P_{r r}= & \frac{f_{\pi}^{2}}{2 \ell^{2}}\left(\eta_{0}^{2}+2 m_{\pi}^{2} \ell^{2}\right) \simeq f_{\pi}^{2} m_{\pi}^{2}, \\
P_{\theta \theta}= & \frac{f_{\pi}^{2}}{2 \ell^{2}}\left(2 m_{\pi}^{2} \ell^{2}(2 \cos \alpha-1)-\eta_{0}^{2}\right) \\
\simeq & f_{\pi}^{2} m_{\pi}^{2}(2 \cos \alpha-1.01), \\
P_{\phi \phi}= & \frac{f_{\pi}^{2}}{2 \ell^{2}}\left(2 m_{\pi}^{2} \ell^{2}(2 \cos \alpha-1)\right. \\
& \left.+2 \sin ^{2}(\alpha)\left(p^{2} \sin ^{2}(q \theta)-q^{2}\right),-\eta_{0}^{2}\right) \\
\simeq & f_{\pi}^{2} m_{\pi}^{2}\left(2 \cos \alpha+\sin ^{2}(\alpha)\left(\sin ^{2}(\theta)-1\right)-1.01\right),
\end{aligned}
$$

where the last equalities are obtained using Eq. (49). The fact that the pressure in a certain region of the $(\theta, \phi)$ plane becomes negative follows from the fact that the system is not static, but stationary, and therefore cavitationlike phenomena are possible. Finally, we note that

$T_{0 \phi}=-\frac{f_{\pi}^{2}}{\ell^{2}} \sin ^{2} \alpha \sin ^{2}(q \theta) p^{2}=-f_{\pi}^{2} m_{\pi}^{2} \sin ^{2} \alpha \sin ^{2}(\theta)$,

where the last equation holds for the values in Eq. (49). The presence of a persistent current means that there is a continuous steady energy transfer in the $\phi$ direction, which is due to the presence of a stationary flow.

\section{B. Topological charge}

The topological charge of the solitonic configuration can be written as

$$
B_{\mathrm{m}}=\frac{\ell^{3}}{24 \pi^{2}} \int d r d \theta d \phi \rho_{\mathrm{m}}
$$

where

$$
\rho_{\mathrm{m}}=\epsilon^{i j k} \operatorname{Tr}\left\{\left(\Sigma^{-1} \partial_{i} \Sigma\right)\left(\Sigma^{-1} \partial_{j} \Sigma\right)\left(\Sigma^{-1} \partial_{k} \Sigma\right)\right\},
$$

is the topological density contribution of the matter fields. The expression in Eq. (59) shows that when the $S U(2)$ valued scalar field $\Sigma$ depends on one or two coordinates, the topological density identically vanishes. Upon substituting Eq. (1) in Eq. (59), the topological density can be rewritten as

$$
\rho_{\mathrm{m}}=-12 \frac{p q}{\ell^{3}} \sin (q \theta) \sin ^{2}(\alpha) \eta,
$$

which can be readily integrated noticing that

$$
\int d r \sin ^{2}(\alpha) \eta=\int d \alpha \sin ^{2} \alpha .
$$

Since we have imposed the boundary condition in Eq. (46), the topological charge becomes

$$
B_{\mathrm{m}}= \begin{cases}-n p & \text { if } q \text { odd } \\ 0 & \text { if } q \text { even }\end{cases}
$$

which clarifies the choice of the $q$ parameter in Eq. (36). We report in Fig. 3 the topological charge densities for the cases with $n=1$ (left panel) and $n=2$ (right panel). Physically, the topological charge represents the baryonic charge of the system $[155,156]$. Therefore, the spatial modulation of the fields is associated with the realization of a nonvanishing baryonic density. With reference to the $q=1$ and $n=2$ case, we see from Eqs. (60) and (61) that the two maxima of the topological charge density correspond to $\theta=\pi / 2, \alpha=\pi / 2$, and $\alpha=3 \pi / 2$. The normal and condensed phases correspond to values of $\alpha$ in the intervals $[0, \pi / 2]$ and $[3 \pi / 2,2 \pi]$ [see Eq. (18)]. These facts 

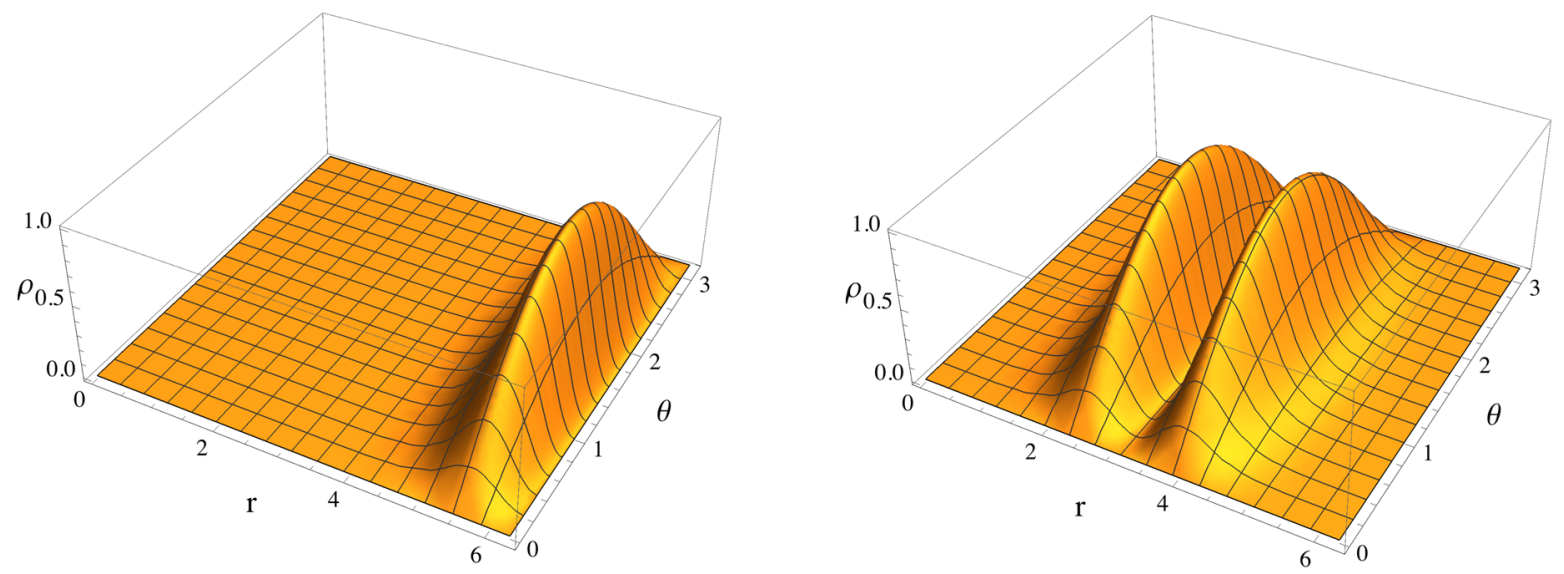

FIG. 3. Modulation of the topological density contribution of the matter fields [see Eq. (60)] obtained for $n=1$ (left-hand-side) and $n=2$ (right-hand-side). The topological density has been normalized to the value at the maximum.

suggest that the spatial modulation of the $\alpha$ field in the interval $[0,2 \pi]$ describes two (anti)baryons, approximately realized in the interval $[\pi / 2,3 \pi / 2]$ and surrounded by a cloud of pions forming a condensate that vanishes at the boundary of the system and reaches its maxima at the places where the baryonic density takes its maximal values. Systems with a larger baryonic charge correspond to larger values of $n$.

\section{INHOMOGENEOUS PHASE INCLUDING THE GAUGE FIELD}

By including the electromagnetic interaction the matter Lagrangian turns to

$$
\begin{aligned}
\mathcal{L}_{\mathrm{m}}= & \frac{f_{\pi}^{2}}{2}\left[\partial_{\mu} \alpha \partial^{\mu} \alpha+\sin ^{2} \alpha \partial_{\mu} \Theta \partial^{\mu} \Theta+2 m_{\pi}^{2} \cos \alpha\right. \\
& \left.+\sin ^{2} \alpha \sin ^{2} \Theta\left(\partial_{\mu} \Phi-2 \tilde{A}_{\mu}\right)\left(\partial^{\mu} \Phi-2 \tilde{A}^{\mu}\right)\right],
\end{aligned}
$$

where $\tilde{A}^{\mu}$ is defined in Eq. (5). From this expression, it is clear that only the $\Phi$ field is minimally coupled to the electromagnetic field. In the following we shall work in the Lorenz gauge, $\partial_{\mu} A^{\mu}=0$; by using the particular classical fields in Eq. (36) and

$$
A^{\mu}=(u, 0,0, u),
$$

the $\alpha(r)$ field decouples and its classical solution satisfies Eq. (39). This means that although the gauge field is generated by the pions, for the particular choice in Eq. (64) it does not back react on the classical fields.

Let us now comment on the particular ansatz in Eq. (64) for the electromagnetic potential. It does not correspond to a particular gauge, but to a particular configuration of the electric and magnetic fields. With this ansatz we have that

$$
E=-\frac{1}{\ell}\left(\partial_{r} u, \partial_{\theta} u, 0\right), \quad B=\frac{1}{\ell}\left(\partial_{\theta} u,-\partial_{r} u, 0\right),
$$

thus the electric and magnetic fields have equal magnitude $|E|^{2}=|B|^{2}=\left(\left(\partial_{r} u\right)^{2}+\left(\partial_{\theta} u\right)^{2}\right) / \ell^{2}$ and they are in the $r-\theta$ plane. The expression of $u$ remains to be determined using the Maxwell equations. From Eq. (8) or Eq. (26) we obtain the electromagnetic current

$$
J_{\mu}=-2 f_{\pi}^{2} \sin ^{2} \alpha \sin ^{2}(q \theta)\left(\partial_{\mu} \Phi-2 \tilde{A}_{\mu}\right),
$$

and therefore the nonvanishing components of the current are

$$
\begin{gathered}
J_{0}=-2 \frac{f_{\pi}^{2}}{\ell} \sin ^{2}(\alpha) \sin ^{2}(q \theta)(p-2 \ell u), \\
J_{\phi}=-J_{0},
\end{gathered}
$$

where we used Eqs. (22) and (38) with $b=1$. Hence, Eq. (7) reduces to the single equation:

$$
\frac{1}{\ell^{2}}\left(\frac{\partial^{2}}{\partial r^{2}}+\frac{\partial^{2}}{\partial \theta^{2}}\right) u=2 \frac{f_{\pi}^{2}}{\ell} \sin ^{2} \alpha \sin ^{2} \Theta(p-2 \ell u),
$$

where we have used Eq. (64) and the Lorenz gauge condition. This expression can be rewritten as a time independent Schrödinger-like equation in a periodic twodimensional potential

$$
\left(\frac{\partial^{2}}{\partial r^{2}}+\frac{\partial^{2}}{\partial \theta^{2}}\right) \Psi+V \Psi=0,
$$

where

$$
\Psi=\frac{p}{\ell}-2 u \quad \text { and } \quad V=4 f_{\pi}^{2} \ell^{2} \sin ^{2} \alpha \sin ^{2}(q \theta),
$$

are the wave function and the effective potential, respectively. As a boundary condition we assume the simplest one, that is, $u=0$ along the whole boundary. The resulting 


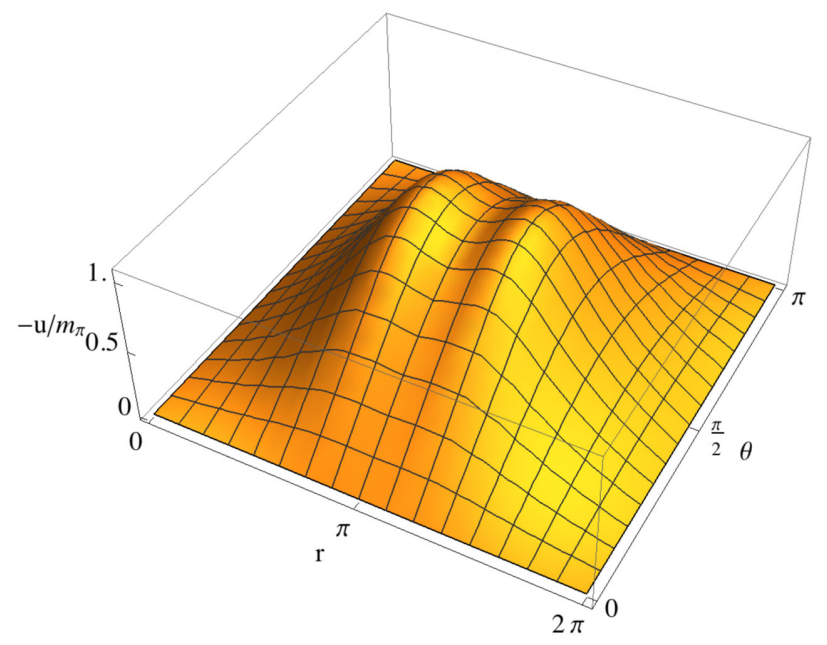

FIG. 4. Modulation of the electromagnetic potential as a function of the $r$ and $\theta$ coordinates obtained by solving the differential equation (70), where $\Psi=1 / \ell-2 u$, assuming that $u=0$ at the boundary.

plot of the potential is in Fig. 4, where we have taken $q=1$ and $p=1$. We tried different boundary conditions with a constant shift of the potential at different boundaries, obtaining similar results.

The corresponding electric and magnetic fields are reported in Fig. 5. The electric field is centered at the maxima of the two solitons and decreases its intensity when approaching the soliton centers. This shows that the electric field is screened inside the solitons. Similarly, the magnetic field in the right panel of Fig. 5 is screened inside the solitons, where the electromagnetic current reaches its maximum (see Fig. 6). Therefore, there is a form of Meissner screening induced by the persistent electromagnetic current $J^{\phi}$ flowing perpendicularly to the $r-\theta$ plane.

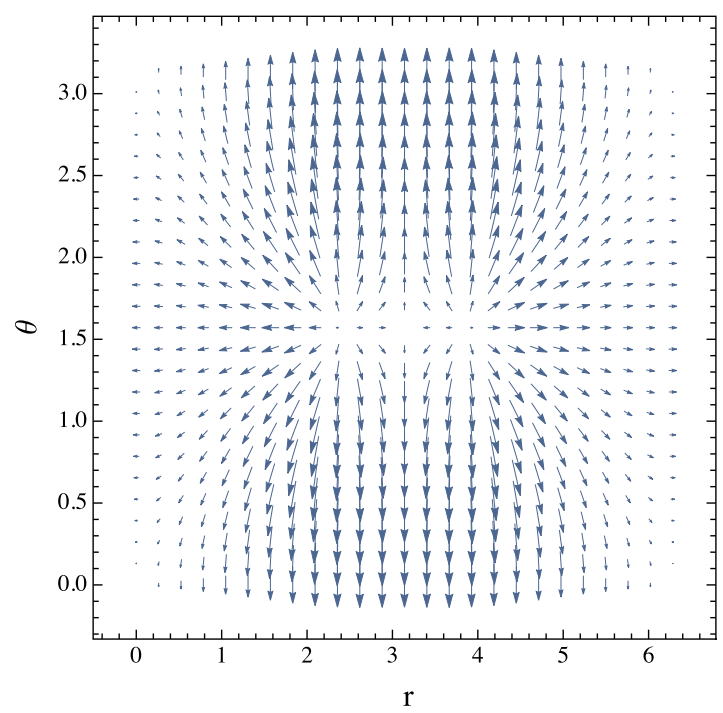

In summary, we have reduced the three coupled field equations in Eq. (6) and the four coupled Maxwell equations in Eq. (7) to the two differential equations in Eqs. (39) and (70). This has been accomplished by the particular ansatz for the angular classical fields in Eq. (36) and the particular choice of the gauge field in Eq. (64). This ansatz greatly simplifies the problem. The linear response of the system to the gauge field, Eq. (66), shows that the produced current has the same form of the energy-density current in Eq. (57), with the additional gauge field contribution.

Indeed, in the presence of the electromagnetic interaction, there are two additional contributions to the energymomentum tensor: one is a contribution determined by the minimal coupling with the $\Phi$ field and the other is the pure gauge one. The minimal coupling can be taken into account using Eq. (51), where now $\Sigma_{\mu}$ is defined in Eq. (3) and includes the gauge field. The pure gauge contribution has the standard expression

$$
T_{\mu \nu}^{\mathrm{em}}=-F_{\mu \alpha} F_{\nu}{ }^{\alpha}+\frac{1}{4} F_{\alpha \beta} F^{\alpha \beta} g_{\mu \nu}
$$

which turns into

$$
T_{\mu \nu}^{\mathrm{em}}=|\boldsymbol{E}|^{2}\left(\begin{array}{cccc}
1 & 0 & 0 & 1 \\
0 & 0 & 0 & 0 \\
0 & 0 & 0 & 0 \\
1 & 0 & 0 & 1
\end{array}\right),
$$

using the particular expression in Eq. (64) of the gauge potential. The energy density now turns into

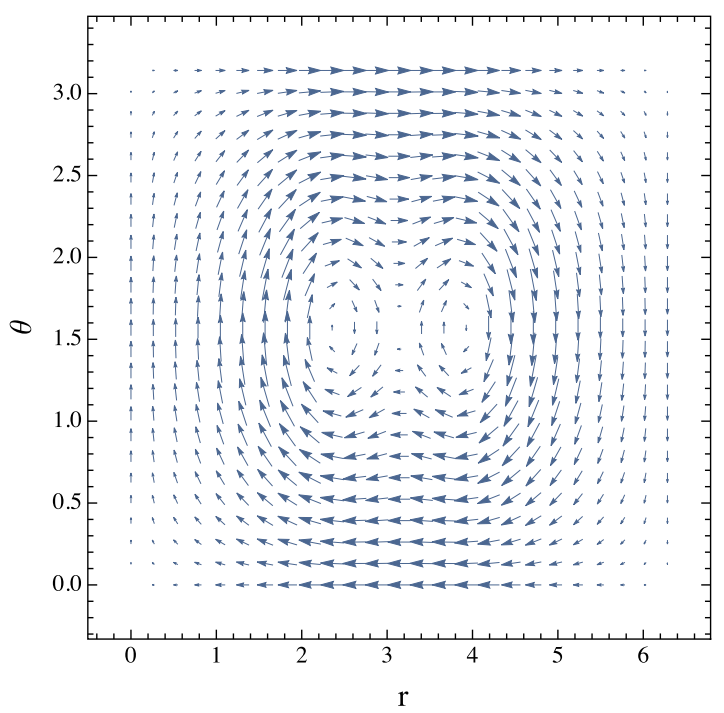

FIG. 5. Electric field (left) and magnetic field (right) obtained with the values in Eq. (49) and assuming that the electromagnetic potential vanishes at the boundary. 


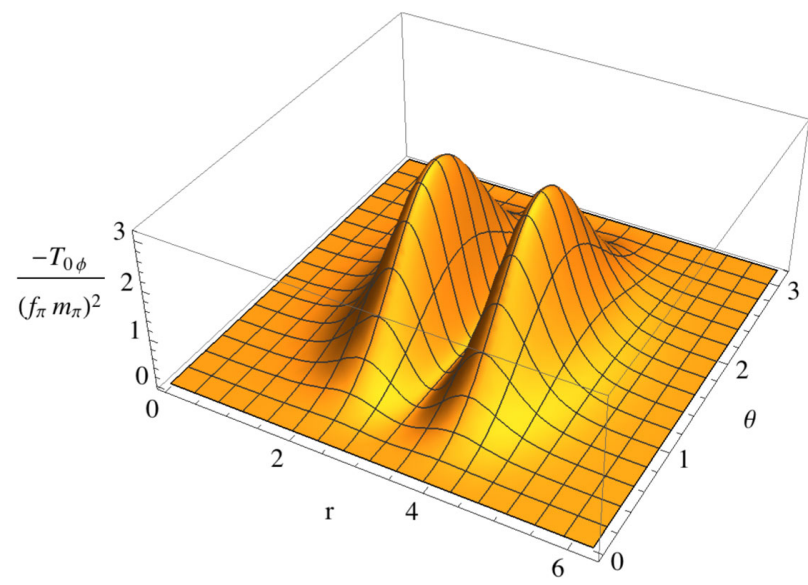

FIG. 6. Intensity of the current $T_{0 \phi}$ defined in Eq. (57) and flowing along the $\phi$ direction orthogonal to the $r-\theta$ plane. The current is concentrated in the regions where the magnetic field reaches its minimum and the topological matter density, as well as the energy density, reach their maximum values.

$$
\begin{aligned}
\epsilon= & \frac{f_{\pi}^{2}}{2 \ell^{2}}\left(\eta^{2}+\left[q^{2}+2(p-2 \ell u)^{2} \sin ^{2}(q \theta)\right] \sin ^{2} \alpha\right. \\
& \left.+2 m_{\pi}^{2} \ell^{2}(1-\cos \alpha)\right)+|E|^{2},
\end{aligned}
$$

and taking into account the ranges in Eq. (23), the total energy of the system is

$$
E=E_{\mathrm{m}}+E_{1}+E_{\mathrm{g}}
$$

where $E_{\mathrm{m}}$ is in Eq. (52) and

$$
E_{1} \simeq 17.5 \frac{f_{\pi}^{2}}{m_{\pi}}, \quad E_{\mathrm{g}} \simeq 2.2 m_{\pi}
$$

are obtained considering $\ell=1 / m_{\pi}$.

The $P_{r r}$ and $P_{\theta \theta}$ pressure components have the same expressions that are reported in Eq. (55), while

$$
\begin{aligned}
P_{\phi \phi}= & \frac{f_{\pi}^{2}}{2 \ell^{2}}\left(2 \ell^{2} m_{\pi}^{2} \cos \alpha-\eta^{2}-q^{2} \sin ^{2} \alpha\right. \\
& \left.+2 \sin ^{2}(q \theta) \sin ^{2}(q \theta)(-p+2 \ell u)^{2}\right)+|E|^{2} .
\end{aligned}
$$

Thus, there are additional gauge field contributions only to the pressure in the $\phi$ direction. As before, the energy density and the pressure are not time dependent; the system is stationary with a constant energy transfer in the $\phi$ direction given by

$$
T_{0 \phi}=-\frac{f_{\pi}^{2}}{\ell} \sin ^{2} \alpha \sin ^{2}(q \theta)(p-2 \ell u)^{2}-|E|^{2},
$$

where the last term on the right hand side is the electromagnetic Poynting vector.
For completeness, we notice that when gauging the $U(1)$ symmetry, one should include an additional contribution to the topological charge $[155,156]$, which now reads

$$
B=\frac{\ell^{3}}{24 \pi^{2}} \int d r d \theta d \phi\left(\rho_{\mathrm{m}}+\rho_{g}\right)=B_{\mathrm{m}}+B_{\mathrm{g}},
$$

where $B_{\mathrm{m}}$ is defined in Eq. (58). The gauge field contribution,

$$
\rho_{g}=-i \epsilon^{i j k} \operatorname{Tr}\left\{\partial_{i}\left[3 A_{j} \sigma_{3}\left(\Sigma^{-1} \partial_{k} \Sigma+\left(\partial_{k} \Sigma\right) \Sigma^{-1}\right)\right]\right\},
$$

is the Callan-Witten topological charge-it guarantees both the conservation and the gauge invariance of the topological charge for the considered potential $A_{j}=\delta_{j 3} u$. Hence, this topological density can be written as

$$
\rho_{g}=-3 i \epsilon^{i 3 k} \partial_{i} u T_{k}=\frac{3 i}{\ell}\left[\partial_{r}\left(u T_{2}\right)-\partial_{\theta}\left(u T_{1}\right)\right],
$$

where

$$
T_{k}=\operatorname{Tr}\left[\sigma_{3}\left(\Sigma^{-1} \partial_{k} \Sigma+\left(\partial_{k} \Sigma\right) \Sigma^{-1}\right)\right],
$$

dictates the dependence on the solitonic field. Then for the considered solitonic configuration we have that

$$
B_{g}=\frac{i \ell^{2}}{4 \pi}\left\{\left.\int_{0}^{\pi} d \theta u T_{2}\right|_{r=0} ^{r=2 \pi}-\left.\int_{0}^{2 \pi} d r u T_{1}\right|_{\theta=0} ^{\theta=\pi}\right\},
$$

where

$$
T_{1}=4 i \frac{\eta}{\ell} \cos \Theta, \quad T_{2}=-2 i \frac{q}{\ell} \sin 2 \alpha \sin \Theta .
$$

Since along the boundary the gauge field takes the fixed value $\bar{u}$, and since $\sin 2 \alpha(r=2 \pi)=\sin 2 \alpha(r=0)=0$ [see Eq. (46)], we readily have that $\left.u T_{2}\right|_{r=0} ^{r=2 \pi}=0$. Then,

$$
B_{g}=\frac{\ell}{\pi} \bar{u}(\cos (q \pi)-1) \int_{0}^{2 \pi} d r \eta .
$$

For $\bar{u}=0$, corresponding to the boundary condition used to obtain Fig. 2, we have that $B_{g}=0$, meaning that there is no contribution of the gauge fields to the topological charge.

For different boundary conditions the topological charge may give a nonvanishing contribution. For instance, for $\bar{u}=p / 2 \ell$, corresponding to vanishing currents at the boundary, we have that

$$
B_{g}= \begin{cases}-n p & \text { if } q \text { odd } \\ 0 & \text { if } q \text { even }\end{cases}
$$

and comparing this with Eq. (62), we find that $B=B_{m}+B_{g}=2 B_{m}=-2 n p$, where the last equality holds for odd $q$. 


\section{CONCLUSIONS AND PERSPECTIVES}

We have derived the first analytic example of topologically nontrivial inhomogeneous pion condensates in lowenergy QCD in $(3+1)$ dimensions at finite isospin chemical potential. We have shown that for a particular condensate ansatz, the complete set of field equations both with and without the minimal coupling to the electromagnetic field can be consistently reduced to integrable equations, where the isospin chemical potential can be related to the system size. In these configurations the topological charge does not vanish and it is deeply related to the stationary properties of the system. We find that the energy density $\epsilon_{\mathrm{m}}$, the topological density $\rho_{\mathrm{m}}$, and the currents $J_{0}, J_{\phi}$, and $T_{0 \phi}$ are constant in the $\phi$ direction while they depend nontrivially on the two spatial coordinates $r$ and $\theta$. The regions of maximal $\rho_{\mathrm{m}}$ are threedimensional tubes of length $2 \pi \ell$ parallel to the $\phi$ direction (the same is true for $\epsilon$ and for the currents), forming a pastalike phase (see [157-159] and references therein for pasta phases in nuclear physics). It is worth emphasizing that the proposed inhomogeneous phase avoids the Derrick's scaling argument for the existence of solitonic solutions because it is realized in a finite volume with appropriate nontrivial boundary conditions. Moreover, it is not static, because the $J_{0}, J_{\phi}$, and $T_{0 \phi}$ currents do not vanish even when the electromagnetic potential vanishes $(u=0)$, and are maximal where $\sin ^{2}(\alpha) \sin ^{2}(q \theta)=1$ [see Eqs. (57) and (67)], rapidly decreasing far from the peaks. Consequently, these currents cannot be turned off continuously-they are topologically protected. Therefore, these stationary currents are actually supercurrents. If the broken $U(1)$ symmetry is global, they correspond to superfluid currents. If the broken $U(1)$ symmetry is gauged, then they correspond to superconducting currents. Indeed, the typical expression of any supercurrent, see for instance [160], is

$$
J \sim \kappa(\nabla \varphi+A),
$$

where $\kappa$ is a constant, $\varphi$ is a phase, and $A$ is the $U(1)$ gauge potential. In the standard settings [160] there is no topological number associated with $\kappa$; the linear stability of the configurations where $\kappa \neq 0$ is established by direct methods (such as linear perturbation theory) and is determined by a control parameter. In the present case, the factor $\sin ^{2}(\alpha) \sin ^{2}(q \theta)$ in the currents [which plays the role of $\kappa$ in Eq. (87)] is instead topologically protected.

As far as we know, these are the first analytic examples of gauged crystal-like structures in low-energy QCD. The realized configuration can be interpreted as consisting of $n$ baryons embedded in an inhomogeneous pion gas, characterized by the modulation of the $\alpha, \Theta$, and $\Phi$ fields. It would be interesting to compare the inhomogeneous and the homogeneous condensates in order to establish which one is more convenient thermodynamically. At a first glance, we could do this explicitly since we can compute the free energy (in a grand canonical ensemble with a chemical potential $\mu_{T}$ associated with the topological charge) both for the homogeneous and for the inhomogeneous condensates. However, the two condensates seem to be realized in different regimes. If we identify the topological charge with the baryonic charge, the inhomogeneous system corresponds to baryons embedded in a pion gas forming a condensate that reaches its maximum values where the baryonic density is larger. On the other hand, the homogeneous phases attainable within our framework correspond to pure pionic systems. Therefore, the two systems correspond to two different grand canonical ensembles. In principle, it is possible to extend chiral perturbation theory including baryons (see for instance [145-147]), however our procedure is different because we have introduced baryons as topological objects in a cloud of pions with a nonvanishing isospin asymmetry.

Remarkably, in the presence of a nonvanishing isospin chemical potential it is possible to realize the solitonic configuration eliminating any time dependence of the condensate, thus the boundary conditions are time independent, as well. This is a clear improvement with respect to the case of vanishing $\mu_{I}$, in which the solitonic configuration has been realized with a time dependent $\Phi$ field and then with time dependent boundary conditions [120-138]. We have found that when the isospin asymmetry is related to the system size by Eq. (38) any time dependence is eliminated, and only in this case is the topologically stable crystalline phase time independent. It would be interesting to compare our results with those of LQCD simulations, which should realize the condition in Eq. (38) by properly modulating the ratio between the lattice size and the isospin chemical potential. It may well be that the LQCD simulations find that a different configuration is energetically favored. Although stable, the solution we obtained may not correspond to the energetically favored solitonic configuration; to derive our results we have indeed employed a number of simplifying assumptions.

We also notice that, in principle, one can use numerical techniques to solve the set of differential equations (27), (28), and (29). The comparison with the outcome of these numerical simulations would be quite interesting, as well. In any case, as we have shown, the analytic solutions are not just of academic interest, as they disclose relevant physical properties of complex structures that may somehow be hidden in the numerical procedures.

Given the nontrivial crystalline structure of our system, it would also be interesting to investigate the spectrum of its low-energy excitations. While our configurations are protected by their topological charge against decay, the presence of nontrivial pole structures at finite momentum might signal strong fluctuation effects (see, e.g., [161] for a discussion on how an inhomogeneous chiral phase is turned into a quantum spin liquid by fluctuations). For the 
modulations considered in this work, we expect at least some of the low-energy excitations to have a phononlike linear dispersion relation (see [96] for a study in a similar scenario), but a more detailed investigation would definitely be of interest, and we plan to come back to this issue in a future work.

We note that we have derived our results employing leading order $\chi \mathrm{PT}$, however the results reported in [136-138], where additional interaction terms have been introduced, strongly suggest that the present construction could be valid even beyond leading order $\chi \mathrm{PT}$.

If the crystals discussed in this work are realized in dense stellar objects, they may have various phenomenological effects on their properties. Since a solitonic crystal supports superfluid and/or superconductive flows, it would manifest in a pastalike structure that influences the thermal transport properties inside the star. Moreover, it should be characterized by a certain rigidity, meaning that deforming the structure should result in a certain energy cost. Thus, for rotating compact stars, it could be relevant for vortex pinning and may be associated with peculiar stellar glitches. Finally, our results suggest that even pion stars, completely made by pions and electrons $[44,162,163]$, could have a rigid crust made by a solitonic crystal. In this case, this phase should manifest not only in peculiar stellar glitches, but that it could also support quadrupolar mass deformations associated with the continuous emission of gravitational waves. We plan to come back on these very interesting issues in a future publication.

\section{ACKNOWLEDGMENTS}

We thank Rob Pisarski for useful discussions. F. C. has been funded by Fondecyt Grants No. 1200022. M. L. and A. V. are funded by FONDECYT post-doctoral Grants No. 3190873 and No. 3200884. The Centro de Estudios Científicos (CECs) is funded by the Chilean Government through the Centers of Excellence Base Financing Program of Conicyt. S. C. has been supported by the MINECO (Spain) under the Projects No. FPA2016-76005-C2-1-P and No. PID2019-105614GB-C21, and by the Grant No. 2017-SGR-929 (Catalonia).
[1] N. Cabibbo and G. Parisi, Phys. Lett. 59B, 67 (1975).

[2] M. Gyulassy, in Structure and Dynamics of Elementary Matter. Proceedings, NATO Advanced Study Institute, Camyuva-Kemer, Turkey, 2003 (Kluwer Academic, Dordrecht, Netherlands, 2004), pp. 159-182.

[3] E. Shuryak, Prog. Part. Nucl. Phys. 62, 48 (2009).

[4] H. Satz, Lect. Notes Phys. 841, 1 (2012).

[5] S. L. Shapiro and S. A. Teukolsky, Black Holes, White Dwarfs, and Neutron Stars: The Physics of Compact Objects (Wiley-VCH, Berlin, 1983).

[6] N. K. Glendenning, Compact Stars: Nuclear Physics, Particle Physics, and General Relativity (Springer-Verlag, New York, 1997).

[7] N. Brambilla et al., Eur. Phys. J. C 74, 2981 (2014).

[8] J. Smit, Cambridge Lect. Notes Phys. 15, 1 (2002).

[9] C. Gattringer and C. B. Lang, Lect. Notes Phys. 788, 1 (2010).

[10] S. Muroya, A. Nakamura, C. Nonaka, and T. Takaishi, Prog. Theor. Phys. 110, 615 (2003).

[11] C. Schmidt, Proc. Sci., LAT2006 (2006) 021.

[12] P. de Forcrand, Proc. Sci., LAT2009 (2009) 010.

[13] O. Philipsen, Prog. Part. Nucl. Phys. 70, 55 (2013).

[14] G. Aarts, J. Phys. Conf. Ser. 706, 022004 (2016).

[15] M. G. Alford, A. Kapustin, and F. Wilczek, Phys. Rev. D 59, 054502 (1999).

[16] J. B. Kogut and D. K. Sinclair, Phys. Rev. D 66, 014508 (2002).

[17] J. B. Kogut and D. K. Sinclair, Phys. Rev. D 66, 034505 (2002).

[18] J. B. Kogut and D. K. Sinclair, Phys. Rev. D 70, 094501 (2004).
[19] S. R. Beane, W. Detmold, T. C. Luu, K. Orginos, M. J. Savage, and A. Torok, Phys. Rev. Lett. 100, 082004 (2008).

[20] W. Detmold, M. J. Savage, A. Torok, S. R. Beane, T. C. Luu, K. Orginos, and A. Parreno, Phys. Rev. D 78, 014507 (2008).

[21] W. Detmold, K. Orginos, M. J. Savage, and A. WalkerLoud, Phys. Rev. D 78, 054514 (2008).

[22] W. Detmold and B. Smigielski, Phys. Rev. D 84, 014508 (2011).

[23] W. Detmold, K. Orginos, and Z. Shi, Phys. Rev. D 86, 054507 (2012).

[24] G. Endrödi, Phys. Rev. D 90, 094501 (2014).

[25] O. Janssen, M. Kieburg, K. Splittorff, J. J. M. Verbaarschot, and S. Zafeiropoulos, Phys. Rev. D 93, 094502 (2016).

[26] B. B. Brandt and G. Endrodi, Proc. Sci., LATTICE2016 (2016) 039.

[27] B. B. Brandt and G. Endrodi, Phys. Rev. D 99, 014518 (2019).

[28] B. B. Brandt, G. Endrodi, and S. Schmalzbauer, EPJ Web Conf. 175, 07020 (2018).

[29] B. B. Brandt, G. Endrodi, and S. Schmalzbauer, Proc. Sci., Confinement2018 (2018) 260.

[30] G. Baym and D. K. Campbell, in Mesons in Nuclei, 1979 (North Holland Publishing Company, 1978), p. 1031.

[31] D. B. Kaplan and A. E. Nelson, Phys. Lett. B 175, 57 (1986).

[32] C. Dominguez, M. Loewe, and J. Rojas, Phys. Lett. B 320, 377 (1994).

[33] D. Son and M. A. Stephanov, Phys. Rev. Lett. 86, 592 (2001).

[34] J. Kogut and D. Toublan, Phys. Rev. D 64, 034007 (2001).

[35] M. C. Birse, T. D. Cohen, and J. A. McGovern, Phys. Lett. B 516, 27 (2001). 
[36] K. Splittorff, D. Toublan, and J. J. M. Verbaarschot, Nucl. Phys. B639, 524 (2002).

[37] M. Loewe and C. Villavicencio, Phys. Rev. D 67, 074034 (2003).

[38] M. Loewe and C. Villavicencio, Phys. Rev. D 70, 074005 (2004).

[39] M. Loewe and C. Villavicencio, arXiv:1107.3859.

[40] A. Mammarella and M. Mannarelli, Phys. Rev. D 92, 085025 (2015).

[41] P. Adhikari, T. D. Cohen, and J. Sakowitz, Phys. Rev. C 91, 045202 (2015).

[42] S. Carignano, A. Mammarella, and M. Mannarelli, Phys. Rev. D 93, 051503 (2016).

[43] M. Loewe, A. Raya, and C. Villavicencio, Phys. Rev. D 95, 096013 (2017).

[44] S. Carignano, L. Lepori, A. Mammarella, M. Mannarelli, and G. Pagliaroli, Eur. Phys. J. A 53, 35 (2017).

[45] P. Adhikari, Phys. Lett. B 790, 211 (2019).

[46] L. Lepori and M. Mannarelli, Phys. Rev. D 99, 096011 (2019).

[47] P. Adhikari, J. O. Andersen, and P. Kneschke, Eur. Phys. J. C 79, 874 (2019).

[48] A. N. Tawfik, A. M. Diab, M. T. Ghoneim, and H. Anwer, Int. J. Mod. Phys. A 34, 1950199 (2019).

[49] I. N. Mishustin, D. V. Anchishkin, L. M. Satarov, O. S. Stashko, and H. Stoecker, Phys. Rev. C 100, 022201 (2019).

[50] P. Adhikari and J. O. Andersen, Phys. Lett. B 804, 135352 (2020).

[51] P. Adhikari and J. O. Andersen, J. High Energy Phys. 06 (2020) 170.

[52] P. Adhikari and H. Nguyen, Eur. Phys. J. Plus 135, 789 (2020).

[53] P. Adhikari and J. O. Andersen, Eur. Phys. J. C 80, 1028 (2020).

[54] N. Agasian, JETP Lett. 111, 201 (2020).

[55] P. Adhikari, J. O. Andersen, and M. A. Mojahed, Eur. Phys. J. C 81, 173 (2021).

[56] A. Barducci, R. Casalbuoni, S. De Curtis, R. Gatto, and G. Pettini, Phys. Rev. D 42, 1757 (1990).

[57] D. Toublan and J. B. Kogut, Phys. Lett. B 564, 212 (2003).

[58] A. Barducci, R. Casalbuoni, G. Pettini, and L. Ravagli, Phys. Rev. D 69, 096004 (2004).

[59] A. Barducci, R. Casalbuoni, G. Pettini, and L. Ravagli, Phys. Rev. D 71, 016011 (2005).

[60] L.-Y. He, M. Jin, and P.-F. Zhuang, Phys. Rev. D 71, 116001 (2005).

[61] D. Ebert and K. G. Klimenko, J. Phys. G 32, 599 (2006).

[62] D. Ebert and K. G. Klimenko, Eur. Phys. J. C 46, 771 (2006).

[63] S. Mukherjee, M. G. Mustafa, and R. Ray, Phys. Rev. D 75, 094015 (2007).

[64] L. He and P. Zhuang, Phys. Lett. B 615, 93 (2005).

[65] L. He, M. Jin, and P. Zhuang, Phys. Rev. D 74, 036005 (2006).

[66] G.-f. Sun, L. He, and P. Zhuang, Phys. Rev. D 75, 096004 (2007).

[67] J. O. Andersen and L. Kyllingstad, J. Phys. G 37, 015003 (2010).
[68] H. Abuki, M. Ciminale, R. Gatto, N. D. Ippolito, G. Nardulli, and M. Ruggieri, Phys. Rev. D 78, 014002 (2008).

[69] H. Abuki, R. Anglani, R. Gatto, M. Pellicoro, and M. Ruggieri, Phys. Rev. D 79, 034032 (2009).

[70] C.-f. Mu, L.-y. He, and Y.-x. Liu, Phys. Rev. D 82, 056006 (2010).

[71] T. Xia, L. He, and P. Zhuang, Phys. Rev. D 88, 056013 (2013).

[72] T. Xia and P. Zhuang, Chin. Phys. C 43, 054103 (2019).

[73] J. Chao, M. Huang, and A. Radzhabov, Chin. Phys. C 44, 034105 (2020).

[74] T. G. Khunjua, K. G. Klimenko, and R. N. Zhokhov, J. High Energy Phys. 06 (2019) 006.

[75] T. Khunjua, K. Klimenko, and R. Zhokhov, Symmetry 11, 778 (2019).

[76] S. S. Avancini, A. Bandyopadhyay, D. C. Duarte, and R. L. S. Farias, Phys. Rev. D 100, 116002 (2019).

[77] Z.-Y. Lu, C.-J. Xia, and M. Ruggieri, Eur. Phys. J. C 80, 46 (2020).

[78] G. Cao and L. He, Phys. Rev. D 100, 094015 (2019).

[79] T. Khunjua, K. Klimenko, and R. Zhokhov, J. High Energy Phys. 06 (2020) 148.

[80] T. Khunjua, K. Klimenko, and R. Zhokhov, Eur. Phys. J. C 80, 995 (2020).

[81] S. Mao, Phys. Rev. D 102, 114006 (2020).

[82] M. Mannarelli, Particles 2, 411 (2019).

[83] K. Fukushima and T. Hatsuda, Rep. Prog. Phys. 74, 014001 (2011).

[84] R. Anglani, R. Casalbuoni, M. Ciminale, N. Ippolito, R. Gatto, M. Mannarelli, and M. Ruggieri, Rev. Mod. Phys. 86, 509 (2014).

[85] M. Buballa and S. Carignano, Prog. Part. Nucl. Phys. 81, 39 (2015).

[86] M. Harada, H. K. Lee, Y.-L. Ma, and M. Rho, Phys. Rev. D 91, 096011 (2015).

[87] V. Kaplunovsky, D. Melnikov, and J. Sonnenschein, Mod. Phys. Lett. B 29, 1540052 (2015).

[88] B.-Y. Park, W.-G. Paeng, and V. Vento, Nucl. Phys. A989, 231 (2019).

[89] G. Basar and G. V. Dunne, Phys. Rev. Lett. 100, 200404 (2008).

[90] G. Basar and G. V. Dunne, Phys. Rev. D 78, 065022 (2008).

[91] G. Basar, G. V. Dunne, and M. Thies, Phys. Rev. D 79, 105012 (2009).

[92] M. Thies, Phys. Rev. D 69, 067703 (2004).

[93] B. Bringoltz, Phys. Rev. D 79, 125006 (2009).

[94] D. Nickel, Phys. Rev. D 80, 074025 (2009).

[95] F. Karbstein and M. Thies, Phys. Rev. D 75, 025003 (2007).

[96] K. Takayama and M. Oka, Nucl. Phys. A551, 637 (1993).

[97] V. Schon and M. Thies, Phys. Rev. D 62, 096002 (2000).

[98] N. Gubina, K. Klimenko, S. Kurbanov, and V. Zhukovsky, Phys. Rev. D 86, 085011 (2012).

[99] J. O. Andersen and P. Kneschke, Phys. Rev. D 97, 076005 (2018).

[100] T. Brauner and N. Yamamoto, J. High Energy Phys. 04 (2017) 132. 
[101] X.-G. Huang, K. Nishimura, and N. Yamamoto, J. High Energy Phys. 02 (2018) 069.

[102] D. J. Gross and A. Neveu, Phys. Rev. D 10, 3235 (1974).

[103] R. F. Dashen, B. Hasslacher, and A. Neveu, Phys. Rev. D 12, 2443 (1975).

[104] S.-S. Shei, Phys. Rev. D 14, 535 (1976).

[105] J. Feinberg and A. Zee, Phys. Rev. D 56, 5050 (1997).

[106] B. J. Schroers, Phys. Lett. B 356, 291 (1995).

[107] K. Arthur and D. H. Tchrakian, Phys. Lett. B 378, 187 (1996).

[108] J. Gladikowski, B. M. A. G. Piette, and B. J. Schroers, Phys. Rev. D 53, 844 (1996).

[109] Y. M. Cho and K. Kimm, Phys. Rev. D 52, 7325 (1995).

[110] A. Yu. Loginov and V. V. Gauzshtein, Phys. Lett. B 784, 112 (2018).

[111] C. Adam, C. Naya, T. Romanczukiewicz, J. SanchezGuillen, and A. Wereszczynski, J. High Energy Phys. 05 (2015) 155.

[112] C. Adam, T. Romanczukiewicz, J. Sanchez-Guillen, and A. Wereszczynski, J. High Energy Phys. 11 (2014) 095.

[113] A. Alonso-Izquierdo, W. G. Fuertes, and J. Mateos Guilarte, J. High Energy Phys. 02 (2015) 139.

[114] S. Chimento, T. Ortin, and A. Ruipérez, J. High Energy Phys. 05 (2018) 107.

[115] G. Baym, B. L. Friman, and G. Grinstein, Nucl. Phys. B210, 193 (1982).

[116] T. H. R. Skyrme, Proc. R. Soc. A 260, 127 (1961).

[117] T. H. R. Skyrme, Proc. R. Soc. A 262, 237 (1961).

[118] T. H. R. Skyrme, Nucl. Phys. 31, 556 (1962).

[119] G. H. Derrick, J. Math. Phys. (N.Y.) 5, 1252 (1964).

[120] F. Canfora and H. Maeda, Phys. Rev. D 87, 084049 (2013).

[121] F. Canfora, Phys. Rev. D 88, 065028 (2013).

[122] S. Chen, Y. Li, and Y. Yang, Phys. Rev. D 89, 025007 (2014).

[123] F. Canfora, F. Correa, and J. Zanelli, Phys. Rev. D 90, 085002 (2014).

[124] F. Canfora, M. Di Mauro, M. A. Kurkov, and A. Naddeo, Eur. Phys. J. C 75, 443 (2015).

[125] E. Ayon-Beato, F. Canfora, and J. Zanelli, Phys. Lett. B 752, 201 (2016).

[126] G. Tallarita and F. Canfora, Nucl. Phys. B921, 394 (2017).

[127] F. Canfora, A. Paliathanasis, T. Taves, and J. Zanelli, Phys. Rev. D 95, 065032 (2017).

[128] A. Giacomini, M. Lagos, J. Oliva, and A. Vera, Phys. Lett. B 783, 193 (2018).

[129] M. Astorino, F. Canfora, M. Lagos, and A. Vera, Phys. Rev. D 97, 124032 (2018).

[130] P. D. Alvarez, F. Canfora, N. Dimakis, and A. Paliathanasis, Phys. Lett. B 773, 401 (2017).

[131] L. Avilés, F. Canfora, N. Dimakis, and D. Hidalgo, Phys. Rev. D 96, 125005 (2017).

[132] F. Canfora, M. Lagos, S. H. Oh, J. Oliva, and A. Vera, Phys. Rev. D 98, 085003 (2018).

[133] F. Canfora, Eur. Phys. J. C 78, 929 (2018).

[134] F. Canfora, N. Dimakis, and A. Paliathanasis, Eur. Phys. J. C 79, 139 (2019).
[135] F. Canfora, S. H. Oh, and A. Vera, Eur. Phys. J. C 79, 485 (2019).

[136] P. D. Alvarez, S. L. Cacciatori, F. Canfora, and B. L. Cerchiai, Phys. Rev. D 101, 125011 (2020).

[137] F. Canfora, M. Lagos, and A. Vera, Eur. Phys. J. C 80, 697 (2020).

[138] F. Canfora, A. Giacomini, M. Lagos, S. H. Oh, and A. Vera, Eur. Phys. J. C 81, 55 (2021).

[139] S. Weinberg, Physica (Amsterdam) 96A, 327 (1979).

[140] J. Gasser and H. Leutwyler, Ann. Phys. (N.Y.) 158, 142 (1984).

[141] H. Georgi, Weak Interactions and Modern Particle Theory (Dover books on Physics, 1984).

[142] H. Leutwyler, Ann. Phys. (N.Y.) 235, 165 (1994).

[143] G. Ecker, Prog. Part. Nucl. Phys. 35, 1 (1995).

[144] H. Leutwyler, Helv. Phys. Acta 70, 275 (1997).

[145] A. Pich, in Probing the standard model of particle interactions. Proceedings, Summer School in Theoretical Physics, NATO Advanced Study Institute, 68th Session, Les Houches, France, 1997. Pt. 1, 2 (Oxford University press, 1998), pp. 949-1049.

[146] S. Scherer, Adv. Nucl. Phys. 27, 277 (2003).

[147] S. Scherer and M. R. Schindler, arXiv:hep-ph/0505265.

[148] J. B. Kogut, M. A. Stephanov, and D. Toublan, Phys. Lett. B 464, 183 (1999).

[149] J. B. Kogut, M. A. Stephanov, D. Toublan, J. J. M. Verbaarschot, and A. Zhitnitsky, Nucl. Phys. B582, 477 (2000).

[150] S. Hands, I. Montvay, S. Morrison, M. Oevers, L. Scorzato, and J. Skullerud, Eur. Phys. J. C 17, 285 (2000).

[151] J. B. Kogut, D. K. Sinclair, S. J. Hands, and S. E. Morrison, Phys. Rev. D 64, 094505 (2001).

[152] T. Brauner, Mod. Phys. Lett. A 21, 559 (2006).

[153] V. V. Braguta, E. M. Ilgenfritz, A. Yu. Kotov, A. V. Molochkov, and A. A. Nikolaev, Phys. Rev. D 94, 114510 (2016).

[154] P. Adhikari, S. B. Beleznay, and M. Mannarelli, Eur. Phys. J. C 78, 441 (2018).

[155] C. G. Callan, Jr. and E. Witten, Nucl. Phys. B239, 161 (1984).

[156] B. M. A. G. Piette and D. H. Tchrakian, Phys. Rev. D 62 , 025020 (2000).

[157] D. G. Ravenhall, C. J. Pethick, and J. R. Wilson, Phys. Rev. Lett. 50, 2066 (1983).

[158] M. Hashimoto, H. Seki, and M. Yamada, Prog. Theor. Phys. 71, 320 (1984).

[159] C. Horowitz, D. Berry, C. Briggs, M. Caplan, A. Cumming, and A. Schneider, Phys. Rev. Lett. 114, 031102 (2015).

[160] E. Witten, Nucl. Phys. B249, 557 (1985).

[161] R. D. Pisarski, A. M. Tsvelik, and S. Valgushev, Phys. Rev. D 102, 016015 (2020).

[162] B. B. Brandt, G. Endrődi, E. S. Fraga, M. Hippert, J. Schaffner-Bielich, and S. Schmalzbauer, Phys. Rev. D 98, 094510 (2018).

[163] J. O. Andersen and P. Kneschke, arXiv:1807.08951. 